\title{
Simulation and Analysis of Influence of Group Delay Distortion on Performance of DBF System
}

\author{
Hao GAO \\ Key Lab of Intelligent Computing and Signal Processing of \\ Ministry of Education, Anhui University \\ Hefei, China
}

\begin{abstract}
Group delay distortion between channels is one of the most important factors which affect the BER (bit error rate) performance of DBF (digital beam forming) System. The basic principles of DBF and the concept of group delay are first introduced, and a linear group delay filter is designed based on the relationship between the minimum phase system and the cepstral coefficients. And then a multi-channel group delay distortion model for simulation in MATLAB software is established. A QPSK system is simulated in multi-channel group delay. Simulation results show that the degradation of BER performance is caused by group delay distortion, and the more serious distortion of group delay, the greater deterioration of BER performance.
\end{abstract}

Keywords-DBF; multi-channel; group delay distortion; BER; simulation

\section{INTRODUCTION}

Digital beam forming (DBF) is a beam forming technology which is achieved by digital methods. It has many advantages, such as the adaptive interference is set to zero automatically, the super-resolution is used for the direction of resolution, and the antenna is automatic calibration and ultralow side lobe is also used. Therefore, DBF is widely used in radar [1] [2] [3], navigation [4] [5], communication [6] and so on. And DBF is used in both the transmission and the receiving channels of the array.

The channels mainly include high frequency amplifier, mixer, intermediate frequency amplifier and $\mathrm{A} / \mathrm{D}$ devices in the processing of array. Because of those analog devices and the active circuit which composed by them inevitably exist amplitude and phase difference, it makes the amplitude and the phase are not the same between transmission and receiving channels. When signals go through the respective channels, the group delay distortion may inconsistent with each signal [7] [8], which leads the waveform of each signal not to match between the rising edge and falling edge, so it has a great effect on DBF. Therefore, it has a very important significance to study the influence of the group delay distortion for DBF error performance.

There are many methods to design group delay filter. In this paper, a linear group delay filter is designed based on the relationship between the minimum phase system and the cepstral coefficients. In order to obtain a better group delay characteristics, and ensure that amplitude characteristic is not distortion at the same time, a kind of all-pass filter is designed

*Corresponding author.

Email address: xhli@ahu.edu.cn (Xiaohui LI).

\author{
Xiaohui LI* \\ Key Lab of Intelligent Computing and Signal Processing of \\ Ministry of Education, Anhui University \\ Hefei, China
}

as a group delay filter to simulate the characteristic of the linear group delay distortion channel. This method is based on the relation between the minimum phase system and cepstral coefficient to solve the denominator coefficients of all-pass filter, so it is very easy to realize for only once inverse Fourier transform.

\section{BASIC PRINCIPLES OF DBF AND CONCEPT OF GROUP DELAY}

\section{A. Basic Principles of DBF}

Beam forming is a spatial filtering processing system, which can form the main beam in a specific direction to receive desired signal, and can restrain the interference signal from other direction.

Assuming that receiving antenna is a uniform linear array of $\mathrm{N}$ element, $\theta$ is the incidence angle of the wave, $\theta_{k}$ is the beam direction at time $k, d$ is the distance of each adjacent array elements, $\lambda$ is the wavelength of signals, and each array element is isotropic, where $\mathrm{W}_{i k}=\left[w_{1 k}, w_{2 k}, L, w_{N k}\right]^{\mathrm{T}}$ is the weight vector at time $k$ and the symbol " $\mathrm{T}$ " denotes the transposition operator. Simply, digital beam former is a multiply-accumulator, which is shown in Fig.1.

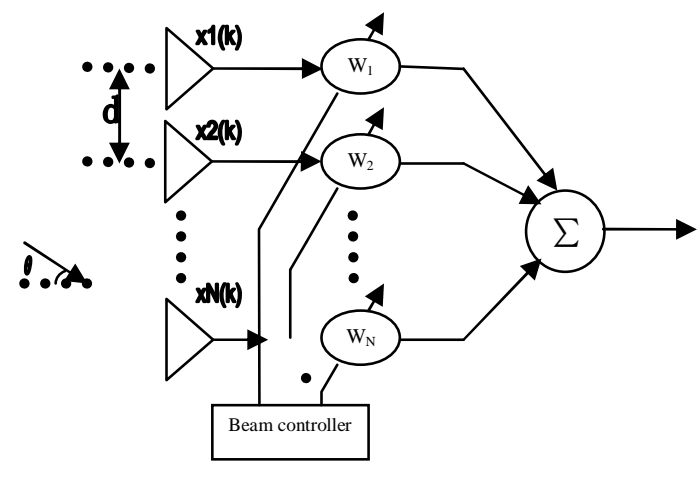

Fig 1 Principle diagram of DBF

The signal $x_{i}$ denotes the complex digital signal which is obtained from the receiving signal after $\mathrm{A} / \mathrm{D}$ and digital orthogonal transformation, where $w_{i k}=a_{i} \exp \left(-j \Delta \phi_{k}\right)$ are the 
channel tap weights, $\Delta \phi_{k}=\frac{2 \pi}{\lambda} d \sin \theta_{k}$ is the phase compensation value, $a_{i}$ is the amplitude weighted coefficient to reduce the antenna side lobe. After compensating the phase and amplitude, the beam synthetic signal can be obtained which is formulated by

$$
y_{k}=\sum_{i=0}^{N-1} w_{i k} x_{i}
$$

So the function of antenna pattern can acquire by modulus (1). Due to the antenna pattern is not the only one, it can change the weight factors to obtain any form of beam. And the signal-to-noise ratio of the output signal can be greatly improved after the digital beam has been synthetic.

\section{B. Concept of Group Delay}

The frequency response function is defined as

$$
H\left(e^{j \omega}\right)=A(\omega) e^{j \theta(\omega)}
$$

Where $\mathrm{A}(\omega)$ is the amplitude-frequency characteristic function, $\theta(\omega)$ is the phase-frequency characteristic function.

The $\tau(\omega)$ is the group delay characteristic function which is defined by

$$
\tau(\omega)=-\frac{d \theta(\omega)}{d \omega}
$$

While $\tau(\omega)$ is a constant, $\theta(\omega)$ is a linear function (for $\theta(\omega)=\omega \tau_{0}$ ), the different frequency components of the signal may have the same group delay. Therefore it will not produce waveform distortion when the signal goes through the channel; otherwise it will cause waveform distortion, and produce the inter-symbol interference, which can lead to the system error performance deteriorative. In addition to the influence of Gaussian noise, the group delay distortion has further deterioration to BER performance in the system.

\section{DESIGN OF GROUP DELAY FILTER}

\section{A. Principle of All-Pass Filter}

Frequency characteristic function is defined as

$$
\mathrm{H}\left(e^{j \omega}\right)=\frac{N(j \omega)}{D(j \omega)}=\frac{\sum_{m=0}^{M} a_{M-m} e^{-j \omega m}}{\sum_{m=0}^{M} a_{m} e^{-j \omega m}}=e^{-j \omega M} \frac{\sum_{m=0}^{M} a_{m} e^{j \omega m}}{\sum_{m=0}^{M} a_{m} e^{-j \omega m}}
$$

Where $a_{0}=1$ is the initial value, from (4), the phase frequency response of filter is obtained by

$$
\theta(\omega)=\theta_{N}(\omega)-\theta_{D}(\omega)=-M \omega-2 \theta_{D}(\omega)
$$

Where $\theta_{N}(\omega)$ is the phase frequency response of the molecular polynomial for system function, while $\theta_{D}(\omega)$ is the denominator polynomial's.
The group delay is acquired as

$$
\tau(\omega)=-\frac{d \theta(\omega)}{d \omega}=M+2 \frac{d \theta_{D}(\omega)}{d \omega}=M-2 \tau_{D}(\omega)
$$

And the relationship between $\tau(\omega)$ and $\tau_{D}(\omega)$ can be written as

$$
\tau_{D}(\omega)=\frac{M-\tau(\omega)}{2}
$$

\section{B. Design of All-Pass Filter}

Where $\tau(\omega)$ is given firstly, and $\tau_{D}(\omega)$ is calculated by (7), then in order to determine the whole filter transfer function, it should calculate the denominator coefficient $a_{m}$.

Denominator characteristic function is defined as

$$
D(\omega)=|D(\omega)| e^{j \theta_{D}(\omega)}
$$

And then equation (8) is taken the natural logarithm as

$$
\ln D(\omega)=\ln |D(\omega)|+j \theta_{D}(\omega)
$$

Because of $D(\omega)$ is a stable minimum phase system, $b(n)$ is its cepstral which is the causal system. So

$$
\begin{aligned}
\ln D(\omega)= & \frac{b(0)}{2}+\sum_{n=1}^{\infty} b(n) e^{-j \omega n}= \\
& \frac{b(0)}{2}+\sum_{n=1}^{\infty} b(n) \cos (\omega n)-j \sum_{n=1}^{\infty} b(n) \sin (\omega n)
\end{aligned}
$$

Comparing (9) to (10), we get the following equation,

$$
\theta_{D}(\omega)=-\sum_{n=1}^{\infty} b(n) \sin (\omega n)+2 k \pi, k=0, \pm 1, \pm 2 \cdots
$$

Equation (11) is derivative on both sides,

$$
a_{0}=1 \sum_{n=0}^{m} \frac{n b(0)=0}{m}
$$

Doing inverse Fourier transform in (12), we can get $b(n)$,

$$
\operatorname{IDFT}\left[\tau_{D}(\omega)\right]=\frac{n b(n)}{2}
$$

Due to the cepstral theory, we get $a_{m}$ by

$$
a_{m}=\sum_{n=0}^{m} \frac{n b(n)}{m} a_{m-n}, m>0
$$

While $b(0)=0$ is the initial value. According to the method above, the linear group delay filter is designed by MATLAB software, and its amplitude characteristics, phase characteristics and the group delay characteristics curves respectively are shown in fig.2, fig.3 and fig.4. 


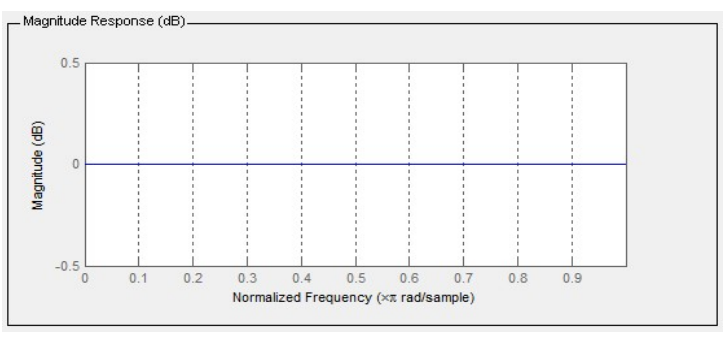

Fig 2 Amplitude characteristic curve of linear group delay filter

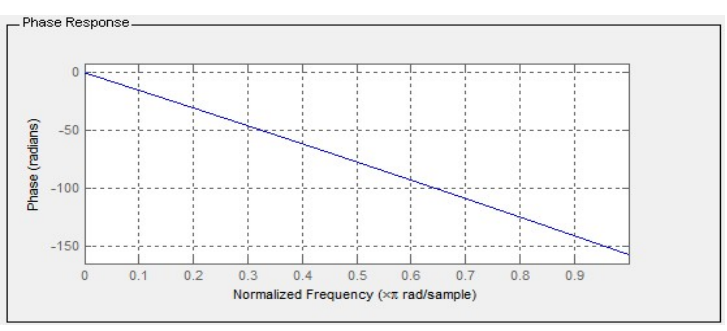

Fig 3 Phase characteristic curve of linear group delay filter

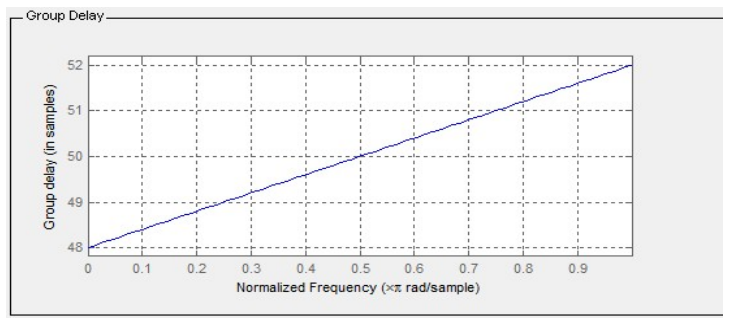

Fig 4 Group delay characteristics curve of linear group delay filter

\section{SIMULATION MODEL AND RESULTS}

It is difficult to use simple analytic expression to indicate the influence of group delay distortion on the system error performance, which brings a certain difficulties in quantitative analysis. So, it is difficult to analysis the influence in theory. But it is a relatively simple method to study this problem through the computer simulation.

Group delay distortion simulation diagram is given firstly in Fig.5, and then a multi-channel group delay distortion model for simulation in MATLAB software is established in Fig.6.

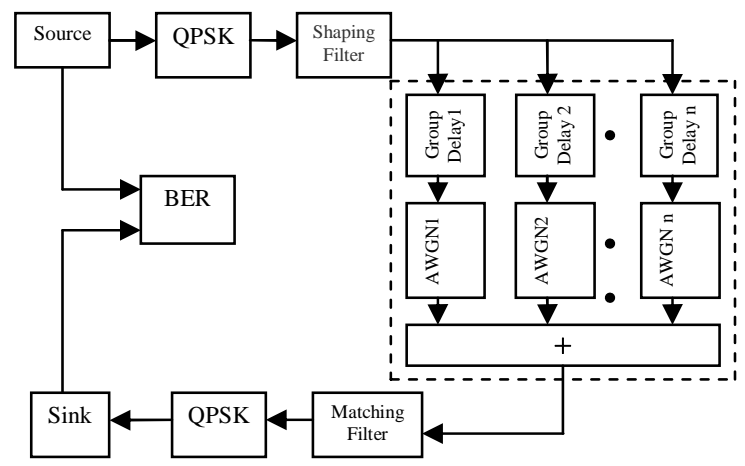

Fig 5 Group delay distortion simulation diagram

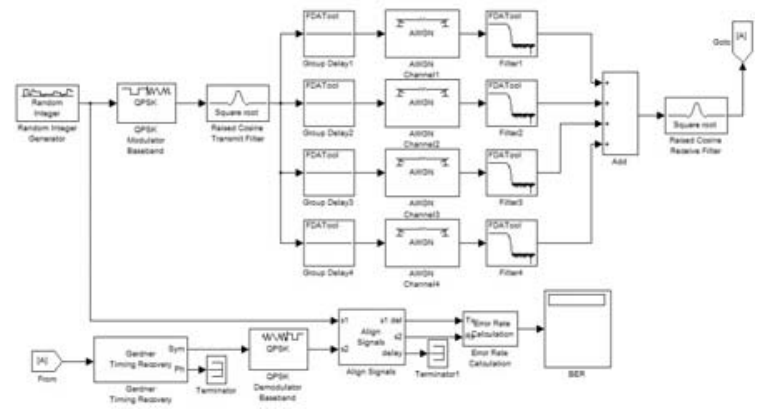

Fig 6 MATLAB simulation model

QPSK is used as a modulation method in system simulation, and the rate of source is $10 \mathrm{Mb} / \mathrm{s}$. The rise cosine square root filter is used in both the transmit side and the receive side with the roll-off coefficient equal to 0.6 , and the filter interpolation is 8 , and the group delay filter is a linear filter which is designed by FDA TOOL [10] in MATLAB software. Noise interference is the white Gaussian noise in the channel.

Ideally, the relationship between error performance and $\mathrm{E}_{b} / N_{0}$ in the coherent demodulation QPSK system is defined as

$$
P_{e}=\frac{1}{2} \operatorname{erfc} \sqrt{\frac{E_{b}}{N_{0}}}
$$

Where $\mathrm{E}_{b}$ is the bit energy of the signal, $N_{0}$ is the power spectrum density of the noise, and $\operatorname{erfc}(x)=\frac{2}{\pi} \int_{0}^{x} e^{-t^{2}} d t$.

4 paths channel is simulated as an example, and two ways are analyzed in the simulation which one has the same maximum group delay distortion and the other is different. Then the BER curves are shown in Fig.7 and Fig.8.

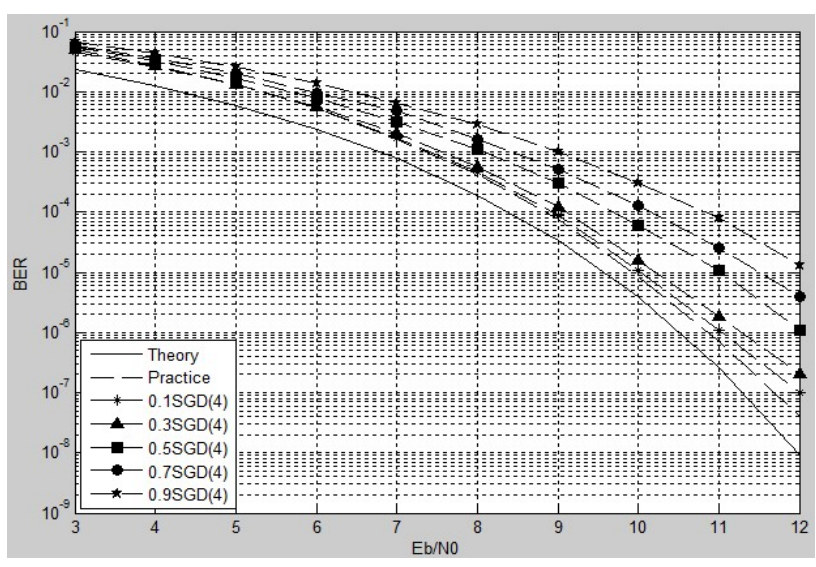

Fig 7 BER curve with the same maximum group delay distortion 


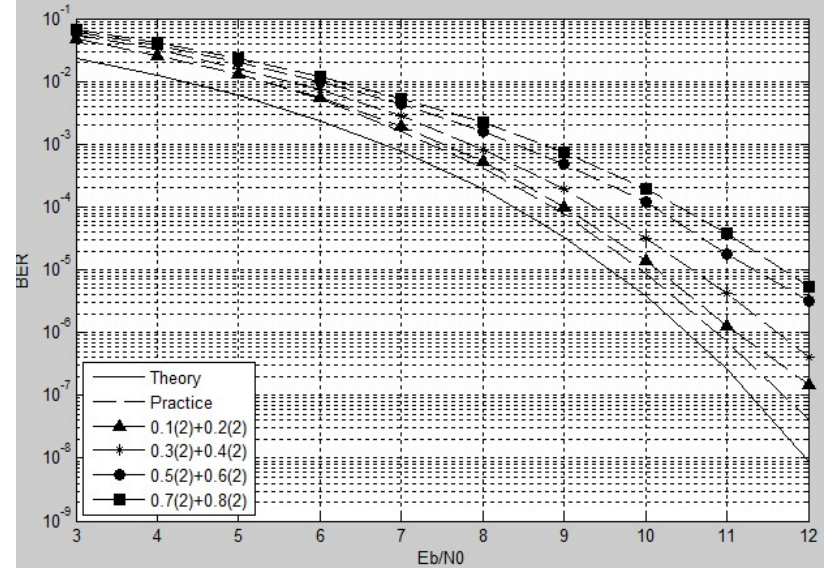

Fig 8 BER curve with the different maximum group delay distortion

In Fig.7, we can see that the bottom curve is the ideal BER curve in Gaussian channel, and the curves in turn up are BER curves with no group delay, and the maximum group delay distortion are respectively 0.1SGD, 0.3SGD, 0.5SGD, 0.7SGD and 0.9SGD. The SGD denotes the symbol number of group delay distortion. And 0.1SGD(4) denotes 4 paths channel which have the same group delay in each path and every path has 0.1 symbol distortion, the rest can be done in the same manner. When the BER is $10^{-5}$, the $\mathrm{E}_{b} / N_{0}$ of the ideal channel is $9.6 \mathrm{~dB}$, the no group delay channel is $9.9 \mathrm{~dB}$, and the others are $10.0 \mathrm{~dB}, 10.3 \mathrm{~dB}, 11.0 \mathrm{~dB}, 11.5 \mathrm{~dB}$ and $12.2 \mathrm{~dB}$, which are respectively deteriorated $0.1 \mathrm{~dB}, 0.4 \mathrm{~dB}, 1.1 \mathrm{~dB}, 1.6 \mathrm{~dB}$ and 2.3dB according to the no group delay channel.

In Fig.8, 0.05(2)+0.1(2) denotes 4 paths which two of them have the same group delay who have 0.05 symbol distortion and the others have the same group delay who have 0.1 symbol distortion, the rest can be done in the same manner. When the BER is $10^{-5}$, the $\mathrm{E}_{b} / N_{0}$ in each of the channels are respectively $10.2 \mathrm{~dB}, 10.6 \mathrm{~dB}, 11.4 \mathrm{~dB}$ and $11.7 \mathrm{~dB}$, which are respectively deteriorated $0.3 \mathrm{~dB}, 0.7 \mathrm{~dB}, 1.5 \mathrm{~dB}$ and $1.8 \mathrm{~dB}$.

\section{Simulation results show that:}

When the group delay distortion is identical in each channel, the BER performance gradually becomes worse with the increasing of the symbols of group delay distortion. When the BER is $10^{-5}$, the deterioration of $\mathrm{E}_{b} / N_{0}$ is $0.1 \mathrm{~dB}$ to $2.3 \mathrm{~dB}$ when the deterioration of the symbol is 0.1SGD to 0.9SGD;

When it is different in each channel, the average group delay distortion is used as a standard, which is acquired by the total numbers of the distortion symbol divided by the total numbers of the channel. The deterioration of $\mathrm{E}_{b} / N_{0}$ is $0.3 \mathrm{~dB}$ to $1.8 \mathrm{~dB}$ when the deterioration of the symbol is $0.15 \mathrm{SGD}$ $((0.1 * 2+0.2 * 2) / 4)$ to $0.75 \operatorname{SGD}((0.7 * 2+0.8 * 2) / 4)$.

And it also shows that the result is the same as the results above if only the average of the group delay distortion is considered. That is the more serious distortion of group delay, the greater deterioration of BER performance. In order to facilitate analysis, the synthetic gain of DBF is not considered in simulation in this paper. There is $6 \mathrm{~dB}$ to improve the synthesis gain for 4 paths channel in theory.

\section{CONCLUSION}

In this paper, we have designed a linear group delay filter which is satisfied with the requirements of simulations, and the filter has been realized based on the relationship between minimum phase system and the cepstral coefficients. The model for simulation has been established, and the simulation has been completed by MATLAB software. The simulation results show that the group delay distortion has a great effect on the BER performance. In order to eliminate the influence, a group delay equalizer should be properly added to ensure that the system can work normally in the actual engineering practice.

\section{ACKNOWLEDGMENT}

This project is supported by the National Natural Science Foundation of China (No. 60972040), the Anhui Provincial Natural Science Foundation (No. 11040606Q06), and the Provincial Project of Natural Science Research for Colleges and Universities of Anhui Province of China (No. KJ2012A003).

\section{REFERENCE}

[1] Huber Sigurd, Younis Marwan, Patyuchenko Anton, et al, "Digital Beam Forming Techniques for Spaceborne Reflector SAR Systems," in the 8th European Conference on EUSAR, 2010, pp.1-4

[2] Sheng-hua Zheng, Hua Han, Da-zhuan Xu, et al, "Evaluation and Measurement of Digital Receiver for DBF Radar," Microwave and Millimeter Wave Technology, vol.4 2004, pp.681-684

[3] Ting Shu, Kai-zhi Wang, Xing-zhao Liu, et al, "Design Considerations for DBF Phased Array 3D Surveillance Radar," in International Conference on CIE Radar (Radar), 2011, pp.360-363

[4] Mei-chao Zhou, "Research on Adaptive Equalization of Group Delay in Satellite Channel," RADIO COMMUNICATIONS TECHNOLOGY, vol.30, 2004, pp.6-8

[5] L. Mohammady, A.R. Eskandari, G. Dadashzadeh, "An Improved Method for Analyzing \& Compensating of Group Delay Variations in Broadband Satellite Signals," in the Fourth Advanced International Conference on Telecommunications, 2008, pp.392-397

[6] Jie Wang, Zhong-hao Zhang, Yue-huan Gong, et al, “An MMSE-DBF Interference Suppression Reception Scheme for MIMO-OSTBCs System," in the 4th International Conference on WiCOM2008, 2008,pp.1-4

[7] Shigeki TAKEDA, Tetsuo ANADA,” Phase Equalizer making use of Negative Group Delay Times by Reflection Coefficients,". Proceedings of the 41st European Microwave Conference, 2011:627-630

[8] A.Azizzadeh, L.Mohammadi, "Degradation of BER by Group Delay in Digital Phase Modulation," in the Fourth International Conference on Telecommunications, 2008, pp.350-354

[9] Ren-fei Zhang, Yu Liu, Xiao-heng Tan, "Simulation and Analysis of the Influence of Multi-channel Group Delay Distortion on the Performance of TDRRSS," Journal of Chongqing University of Posts and Telecommunications, vol.23, 2011, pp.32-37

[10] Lei Ding, Zhen-cun Pan, Wei Cong, "Design and Simulation of Digital Filter Based on Signal Processing Toolbox of MATLAB," RELAY, vol.31, 2003, pp.49-51

[11] Xiang-wei Zhu, Yuan-ling Li, Shao-wei Yong, et al, "A Novel Definition and Measurement Method of Group and Its Application," IEEE Trans on M, vol. 58, 2009, pp.229-233 